\title{
Efficacy and Safety of an Aerosol Box for Percutaneous Tracheostomy in Patients with COVID-19 in an Intensive Care Unit
}

\author{
Mesut Erbas ${ }^{1}$ and Burhan Dost ${ }^{2}$ \\ ${ }^{1}$ Department of Anaesthesiology and Reanimation, Faculty of Medicine, Çanakkale University, Çanakkale, Turkey \\ ${ }^{2}$ Department of Anesthesiology and Reanimation, Faculty of Medicine, Ondokuz Mayis University, Samsun, Turkey
}

\begin{abstract}
Objective: To assess whether the use of an aerosol box for percutaneous tracheostomy in patients with COVID-19 can protect healthcare workers and affect the procedure-related outcomes and complications.

Study Design: Descriptive study.

Place and Duration of Study: Çanakkale Onsekiz Mart University Hospital, Çanakkale, Turkey from March to August, 2020.

Methodology: A retrospective evaluation of patients, who underwent percutaneous tracheostomy with an aerosol box, was conducted. Patients aged over 18 years, diagnosed with COVID-19 and requiring percutaneous tracheostomy were included. Exclusion criteria were: age under 18 years and refusal to participate in the study. Patients' age, gender, comorbidities, APACHE II scores at intensive care admission, numbers of intubated days, durations of tracheostomy opening time, and complications were recorded.

Results: Twenty-four patients underwent the procedure. Tracheostomy was performed successfully in all 24 cases (100\%). The mean age of the cases was $67.2 \pm 10.1$ years. Four patients experienced minor bleeding related to performing the percutaneous tracheostomy using the aerosol box. No healthcare workers were infected with SARS-CoV-2 while performing the tracheostomy procedure.

Conclusion: Use of the aerosol box had adequate efficacy and safety for performance of the percutaneous tracheostomy procedure in intubated patients with COVID-19, who were being followed up in the intensive care unit. This procedure could be used as an alternative to traditional tracheostomy methods in patients with contagious respiratory infections.
\end{abstract}

Key Words: Tracheostomy, Intensive care unit, COVID-19, Aerosol box.

How to cite this article: Erbas M, Dost B. Efficacy and Safety of an Aerosol Box for Percutaneous Tracheostomy in Patients with COVID-19 in an Intensive Care Unit. J Coll Physicians Surg Pak 2021; 31(JCPSPCR):CR79-CR82.

\section{INTRODUCTION}

The coronavirus disease 2019 (COVID-19) pandemic has become a serious global health problem. The clinical symptoms of COVID-19 infection range from asymptomatic disease or mild upper respiratory tract infection to severe viral pneumonia, accompanied by respiratory failure, with the potential for death of the patient. Asignificant number of these patients, therefore, require intensive care and mechanical ventilation. However, patients with critical diseases requiring invasive ventilation frequently run a high risk of mortality of up to $50 \%$, while those who survive their disease often need long-term support. ${ }^{1-4}$

Correspondence to: Dr. Burhan Dost, Department of Anesthesiology and Reanimation, Faculty of Medicine,

Ondokuz Mayis University Samsun, Turkey

E-mail: burhandost@hotmail.com

Received: November 01, 2020; Revised: February 23, 2021; Accepted: March 10, 2021

DOI: https://doi.org/10.29271/jcpsp.2021.JCPSPCR.CR79
Even before the COVID-19 pandemic, patients who underwent endotracheal intubation in the intensive care unit subsequently required long-term mechanical ventilation. In those patients, a percutaneous tracheostomy is typically performed to reduce the complications that may develop due to prolonged endotracheal intubation. ${ }^{5}$ However, with the COVID-19 pandemic, tracheostomy has now become a high-risk procedure for healthcare workers (HCWs), who already face fears of personal infection due to the shortage of personal protective equipment (PPE). ${ }^{6}$

COVID-19 infection is mainly spread by way of droplets, thereby necessitating the use of different safety methods for situations likely to generate aerosols, such as intubation and tracheostomy. Canelli et al. suggested the usefulness of an aerosol box for protection of the operator during tracheal intubation. ${ }^{7}$ An aerosol box typically consists of a transparent polycarbonate cube that covers the head and shoulders of the patient while still providing access to the patient. The type of aerosol box we use for tracheostomy has two more holes on both sides, in addition to the access holes available for the intubation procedure (Figure 1). 


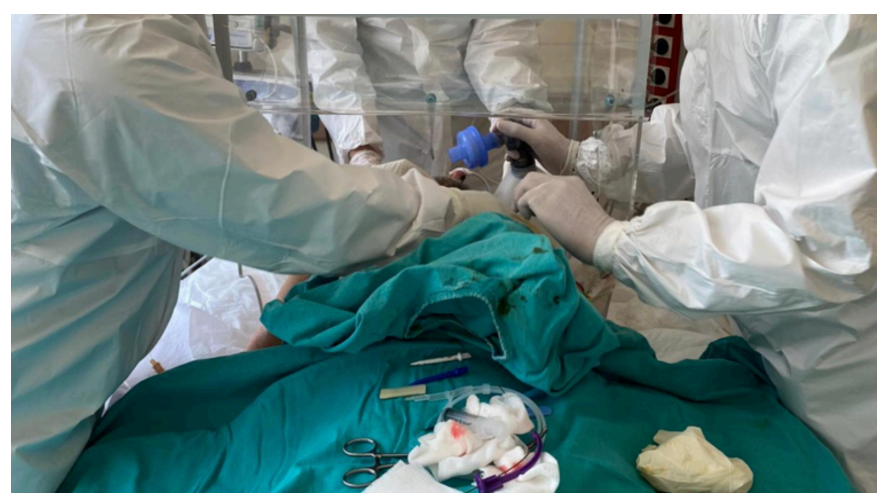

Figure 1: Demonstration of procedure using the aerosol box.

Due to the high mortality associated with COVID-19, and concerns about transmission of the infection, the HCWs are now reluctant to perform open percutaneous tracheostomy in patients with COVID-19.

The aim of this study was to evaluate the effectiveness of an aerosol box for reducing the complications associated with percutaneous tracheostomy and the transmission of COVID-19 to HCWs.

\section{METHODOLOGY}

The study was approved by the University Ethics Committee (June 03, 2020 - Protocol No. 34/08). This was a retrospective evaluation of the patients with COVID-19, who underwent percutaneous tracheostomy from March to August 2020 in the pandemic intensive care unit (ICU) of Çanakkale Onsekiz Mart University Hospital. Patients aged over 18 years, diagnosed with COVID-19 and requiring percutaneous tracheostomy, were included. Exclusion criteria were age under 18 years and refusal to participate in the study.

The collected data included the patients' age, gender, comorbidities, acute physiology and chronic health evaluation (APACHE II) scores at ICU admission, numbers of intubated days, durations of tracheostomy opening time, and complications developing during and after the procedure. Bleeding was categorised as minor bleeding (that stops in a short time with a sponge wrapped around the stoma), moderate bleeding (continuous bleeding coming from the stoma/aspiration trachea despite compression) and abundant bleeding (requiring intervention in the operating room). Complete PPE gear (FFP3 mask, fluid-repellent disposable surgical gown, cap, eye protection and gloves) was worn by all HCWs in the room during the procedure. None of the HCWs involved in performing percutaneous tracheostomies in COVID-19 patients in the ICU developed any clinical COVID-19 symptoms, nor were any diagnosed with COVID-19.

All tracheostomies were performed by the same anaesthesiologist at the patients' bedside in the ICU. The thyroid, cricoid cartilage and vocal cords were first visualised through ultrasonography (USG) on the midline. The USG probe was then held to point to the midline, and the cartilaginous rings of the trachea were visualised by USG scanning starting from the midline in the sagittal plane to the lateral USG. The second and third cartilaginous rings, where the tracheostomy would be performed, were identified and the space between them was marked as the incision and needle- entry sites. Vascular structures at the location selected as the needle-entry point in the midline of the neck were scanned in the transverse plane. If a vascular formation was detected at the needle- entry site, the lower or upper cartilaginous ring gap was examined, and the point where no vascular formation was observed was determined and marked as the needle-entry site. The location of the endotracheal tube was imaged. Before the intervention, $0.6 \mathrm{mg} / \mathrm{Kg}$ rocuronium was used as a myorelaxant and $0.25 \mathrm{mg} / \mathrm{Kg}$ propofol was used as sedative agent. The aerosol box was then placed in the patient's head area (Figure 1). The intubation tube was pulled back up to the vocal cords with the help of a laryngoscope.

During the tracheostomy procedure, ventilation of the patient was maintained. After administering local anaesthesia and making the incision at the point marked with USG, the percutaneous tracheostomy needle was inserted from the marked point, and the procedure was performed under continuous aspiration. The plastic cannula outside the needle was pushed into the trachea, over the needle when the air aspiration was initiated. Then needle was withdrawn and the guide wire was advanced through the plastic cannula and placed in the trachea. The entry point was then dilated to an appropriate extent, first using the plastic dilators and then the dilator clamp over the guide wire. A tracheostomy cannula of a size suitable for the patient was advanced over the guide wire and placed in the trachea. The cannula was fixed after confirming its location in the trachea by auscultation. The ventilator was then connected and restarted once the circuit was closed.

Descriptive statistics were calculated, including means, standard deviations, medians, minimums, maximums, frequencies and ratios. SPSS version 22 software (IBM, Armonk, NY, USA) was used.

\section{RESULTS}

In total, 24 patients with COVID-19 (mean age: $67.2 \pm 10.1$ years, females: $33.3 \%$ ) who underwent percutaneous tracheostomy using an aerosol box, were included in the study. The demographic data of the patients are presented in Table I. Eight patients $(33.3 \%)$ had cardiovascular disease, six (25\%) had respiratory disease, six (25\%) had diabetes mellitus, and four $(16.7 \%)$ had more than one comorbidities. Four patients (16.7\%) experienced minor bleeding related to performing the percutaneous tracheostomy, using the aerosol box. Subcutaneous emphysema occurred in two patients (8.3\%). No healthcare workers were infected with SARS-COV-2, while performing the tracheostomy procedure. Tracheostomy was closed in six patients $(25 \%)$, six patients $(25 \%)$ were discharged from the hospital with a mechanical ventilator, eight patients (33.3\%) remained hospitalised, and four patients (16.7\%) died. 
Table I: Demographic data of the patients.

\begin{tabular}{|c|c|c|c|}
\hline Characteristics & Range (min-max) & Median & Mean \pm SD or $n$ and $\%$ \\
\hline Age (years) & $54-85$ & 66 & $67.2 \pm 10.1$ \\
\hline Sex F/M & & & $8(33.3 \%) / 16(66.7 \%)$ \\
\hline Days intubated before tracheostomy & $12-15$ & 14 & $13.5 \pm 1.3$ \\
\hline APACHE II score at ICU admission & $8.8-16.4$ & 14 & $12.7 \pm 2.5$ \\
\hline Duration of tracheostomy opening time (min) & $10-16$ & 14 & $13.4 \pm 2.2$ \\
\hline
\end{tabular}

\section{DISCUSSION}

This study has shown that the use of an aerosol box during the percutaneous tracheostomy procedure of intubated patients with COVID-19 was adequate in terms of both efficacy and safety. For this reason, this procedure can be used as an alternative method to traditional tracheostomy in patients followed up in the ICU. Tracheostomy is performed to reduce the complications that may develop due to prolonged endotracheal intubation. The overall aims of tracheostomy are to reduce laryngeal damage, to facilitate nursing care and aspiration of the respiratory tract, to increase the patient's mobility by providing a safe airway, to facilitate the transfer of the patient from the ICU, to improve the patient's comfort and to ease oral nutrition. In addition to maintaining the airway and preventing complications related to intubation in the ICU, a tracheostomy also decreases dead space volume, reduces airway resistance and facilitates weaning from ventilation. ${ }^{8}$ Furthermore, studies have demonstrated that pronounced laryngeal oedema and laryngeal ulcerations occur in patients with COVID-19. ${ }^{9}$ In the post-intubation period, coronaviruses are known to cause laryngeal oedema and laryngitis, and SARS-CoV-2 is also thought to have additional direct laryngeal effects in some individuals. ${ }^{10,11}$

The optimal timing of tracheostomy in a critical patient, who is followed up in the ICU during the COVID-19 pandemic is uncertain. ${ }^{12,13}$ Postponing tracheostomy for patients with the SARS-CoV-2 virus can reduce the risk of infection for the HCWs, but this risk reduction needs to be offset by considering the complications of long-term trans-laryngeal tracheal intubation in the patient. The current case series, evidence and expert opinions, when used as guidelines, suggest that a tracheostomy should be postponed until at least the tenth day of mechanical ventilation. In this case, tracheostomy was scheduled for after the tenth day. In their study, Friedman et al. ${ }^{14}$ found a duration of 8.2 minutes for a percutaneous tracheostomy intervention. They also reported that the use of fibre optics, during the procedure, helped reduce complications such as wrong passage and tracheal damage. In this study, the average procedure time was 14.2 minutes.

A percutaneous tracheostomy study conducted on 200 patients by Rajajee et al. ${ }^{15}$ in 2015 compared 107 percutaneous tracheostomy procedures accompanied with USG to 93 procedures conducted without USG. A rate of $1 \%$ was reported for early and late complications developing in patients, who underwent the procedure performed with USG; whereas, this rate was $10 \%$ when the procedure was performed without USG. A reduction in complications with the use of USG before the procedure was also observed, even though the authors were working in a restricted area during the percutaneous tracheostomy and personal protective equipment limited their movements.

It was observed that the formation of a barrier between the patient and the physician during the procedure created confidence in the person who performed the tracheostomy. Nevertheless, the limited range of movements and the use of personal protective equipment extended the duration of the procedure beyond the expected average time, and narrowed the field of view. However, the success of percutaneous tracheostomy in patients with COVID-19 in the ICU is affected by many other factors, such as the experience of the person performing the procedure, the general condition of the patient, and the neck anatomy.

This study has a few limitations. First, it is a retrospective research design, and second it has a small sample size.

\section{CONCLUSION}

The use of the aerosol box had adequate efficacy and safety for the performance of the percutaneous tracheostomy procedure in intubated patients with COVID-19, who were being followed up in the intensive care unit. This procedure could be used as an alternative to traditional tracheostomy methods in patients with contagious respiratory infections.

\section{ETHICAL APPROVAL:}

The study was approved by the University Ethics Committee (June 03, 2020 - Protocol No. 34/08).

\section{PATIENTS' CONSENT:}

Consent for the participation in the study was not obtained from patients as data were collected from the medical record without disclosing participants' identities.

\section{CONFLICT OF INTEREST:}

The authors declared no conflict of interest.

\section{AUTHORS' CONTRIBUTION:}

ME: Conceptualisation, methodology, software, data collection.

ME, BD: Writing the original draft, visualisation, investigation, software, validation reviewing and editing.

\section{REFERENCES}


1. Zhou F, Yu T, Du R, Fan G, Liu Y, Liu Z, et al. Clinical course and risk factors for mortality of adult inpatients with COVID-19 in Wuhan, China: A retrospective cohort study. Lancet 2020; 395(10229):1054-62. doi:10.1016/s01406736(20)30566-3.

2. Dost B, Koksal E, Terzi Ö, Bilgin S, Ustun YB, Arslan HN. Attitudes of anesthesiology specialists and residents toward patients infected with the novel coronavirus (COVID-19): A national survey study. Surg Infect (Larchmt) 2020; 21(4):350-6. doi:10.1089/sur.2020.097.

3. Wang D, Hu B, Hu C, Zhu F, Liu X, Zhang J, et al. Clinical characteristics of 138 hospitalised patients with 2019 novel coronavirus-infected pneumonia in Wuhan, China. Jama 2020; 323(11):1061-1069. doi:10.1001/jama.2020.1585.

4. Chen HHX, Chen S, Zhu W, Huang W, Chen K. Coronavirus disease 2019 (COVID-19) mechanical ventilation research theme analysis: Co-word cluster analysis. J Coll Physicians Surg Pak 2020; 30(10):S94-S100. doi: 10.29271/jcpsp.2020.supp2.94.

5. Yıldırım F, Güllü YT, Demirel CB. Percutaneous tracheostomy in the intensive care unit. Eurasian J Pulmonol 2015; 17:136-141.

6. Duggan LV, Marshall SD, Scott J, Brindley PG, Grocott HP. The MacGyver bias and attraction of homemade devices in healthcare. Can J Anaesth 2019; 66(7):757-61. doi:10.1007/ s12630-019-01361-4.

7. Canelli R, Connor CW, Gonzalez M, Nozari A, Ortega R. Barrier Enclosure during Endotracheal Intubation. N Engl J Med 2020; 382(20):1957-8. doi:10.1056/NEJMc2007589.

8. Kırca H, Çakın Ö, Cengiz MS, Ramazanoğlu A. Tracheotomy in the intensive care unit: Indications, Complications and Prognosis. Türk Yoğun Bakım Dergisi 2018; 16:17-25. doi:10.4274/tybd.03064.

9. McGrath BA, Wallace S, Goswamy J. Laryngeal oedema associated with COVID-19 complicating airway management. Anaesthesia 2020; 75(7):972. doi:10.1111/anae.15092.

10. Brodsky MB, Levy MJ, Jedlanek E, Pandian V, Blackford B, Price $C$, et al. Laryngeal Injury and upper airway symptoms after oral endotracheal intubation with mechanical ventilation during critical care: A systematic review. Crit Care Med 2018; 46(12):2010-2017. doi:10.1097/ccm.000000000000 3368.

11. Lake MA. What we know so far: COVID-19 current clinical knowledge and research. Clin Med (Lond) 2020; 20(2):124-7. doi:10.7861/clinmed.2019-coron.

12. Rumbak MJ, Graves AE, Scott MP, Sporn GK, Walsh FW, Anderson WM, et al. Tracheostomy tube occlusion protocol predicts significant tracheal obstruction to air flow in patients requiring prolonged mechanical ventilation. Crit Care Med 1997; 25(3):413-7. doi:10.1097/ 00003246-199703000-00007.

13. McGrath BA, Ashby N, Birchall M, Dean P, Doherty C, Ferguson $\mathrm{K}$, et al. Multidisciplinary guidance for safe tracheostomy care during the COVID-19 pandemic: The NHS national patient safety improvement programme (NatPatSIP). Anaesthesia 2020; 75(12):1659-70. doi:10.1111/anae. 15120.

14. Friedman Y, Fildes J, Mizock B, Samuel J, Petal S, AppavuMD $\mathrm{S}$, et al. Comparison of percutaneous and surgical tracheostomies. Chest 1996; 110(2):480-5. doi:10.1378/chest. 110.2.480.

15. Rajajee V, Williamson CA, West BT. Impact of real-time ultrasound guidance on complications of percutaneous dilatational tracheostomy: A propensity score analysis. Crit Care 2015; 19(1):198. doi:10.1186/s13054-015-0924-7. 